\title{
RAYLEIGH-BÈNARD CONVECTION IN A SECOND-ORDER FLUID WITH MAXWELL-CATTANEO LAW
}

\author{
Smita. Saklesh. Nagouda* and S. Pranesh \\ Department of Mathematics, Christ University, Hosur Road, Bangalore-560029, India \\ smita.nagouda@christuniversity.in \\ pranesh.s@christuniversity.in \\ ${ }^{*}$ Corresponding Author
} Keywords: Rayleigh-Bènard convection, Maxwell-Cattaneo law, Second order fluid, Galerkin
Method, Biot number

\begin{abstract}
The objective of the paper is to study the Rayleigh-Bènard convection in second order fluid by replacing the classical Fourier heat law by non-classical Maxwell-Cattaneo law using Galerkin technique. The eigen value of the problem is obtained using the general boundary conditions on velocity and third type of boundary conditions on temperature. A linear stability analysis is performed. The influence of various parameters on the onset of convection has been analyzed. The classical Fourier flux law over predicts the critical Rayleigh number compared to that predicted by the non-classical law. The present non-classical Maxwell-Cattaneo heat flux law involves a wave type heat transport(SECOND SOUND) and does not suffer from the physically unacceptable drawback of infinite heat propagation speed. It is found that the results are noteworthy at short times and the critical eigen values are less than the classical ones. Over stability is the preferred mode of convection.
\end{abstract}

\section{INTRODUCTION}

Viscoelastic fluids when modeled by the Coleman-Noll constitutive equation is termed as second-order fluids. Second-order fluids are dilute polymeric solutions(e.g., poly-ios-butylene in Cetane, Methyl-Methacrylate in n-butyl acetate, polyethylene oxide in water, etc.). These fluids express stress in terms of a second degree polynomial in rate of strain. Coleman and

Noll [1] have studied, for the first time a simple fluid having fading memory and suggested a second order correction to incompressible perfect fluids. Coleman and Noll [1] derived the following constitutive equation for an incompressible second-order fluid;

$$
\tilde{\tau}=-\mathrm{pI}+\mu_{1} \widetilde{\mathrm{A}}_{(1)}+\mu_{2} \widetilde{\mathrm{A}}_{(2)}+\mu_{3} \widetilde{\mathrm{A}}_{(1)}^{2}
$$

Where $\tilde{i}$ is the stress tensor, $\mathrm{p}$ is an indeterminate pressure, $\mu_{1}, \mu_{2}$ and $\mu_{3}$ are measurable material constants and they denote respectively viscosity, elasticity and cross viscosity. These materials can be determined from viscometric flows for any real fluid. $\mathbf{A}_{(\mathbf{1})}$ and $\mathbf{A}_{(2)}$ are Rivlin- Ericken tensors and they denote respectively the rate of strain and acceleration. Kaloni [2] has made some useful remarks on the parameters and provided useful theorems for Second-Order Fluids. Siddheshwar and Srikrishna [3] have studied linear and non-linear convection in second-order fluid. They found that in the linear theory the critical eigen value is independent of viscoelastic effects and principle of exchange of stability holds. An autonomous system of differential equations representing cellular convection arising in the non-linear study is solved numerically. Recently Siddeshwar et al [4], Sekhar and Jaylatha [5] have studied the Rayleigh Benard situation in Second order fluid. The reported works on convection are with classical Fourier heat flux law.

The Classical Fourier law of heat conduction expresses the heat flux within a medium is proportional to the local temperature gradient in the system. A well known consequence of this law is that heat perturbations propagate with an infinite velocity. This drawback of the classical law motivated Lindsay and Stranghan [6], Stranghan and Franchi [7], Lebon and Cloot [8] and Pranesh and Siddheshwar [9, 10], Pranesh and Kiran [11] to adopt a non-classical heat flux law in studying Rayleigh-Bènard / Marangoni convection. The hyperbolic heat equation (SECOND SOUND) 
model adopted by these authors does not suffer from the physically unacceptable drawback of infinite heat propagation speed. The principle of exchange of stability is also not guaranteed in these problems.

The objective of this problem is to replace the classical parabolic heat equations by non-classical Maxwell-Cattaneo heat flux law and study Rayleigh-Bènard convection in second-order fluids.

\section{MATHEMATICAL FORMULATION:}

Consider a horizontal layer of infinite extent occupied by a Second order fluid of depth $\mathrm{d}$. Let $\Delta \mathrm{T}$ be the temperature difference between lower and upper fluid surfaces.

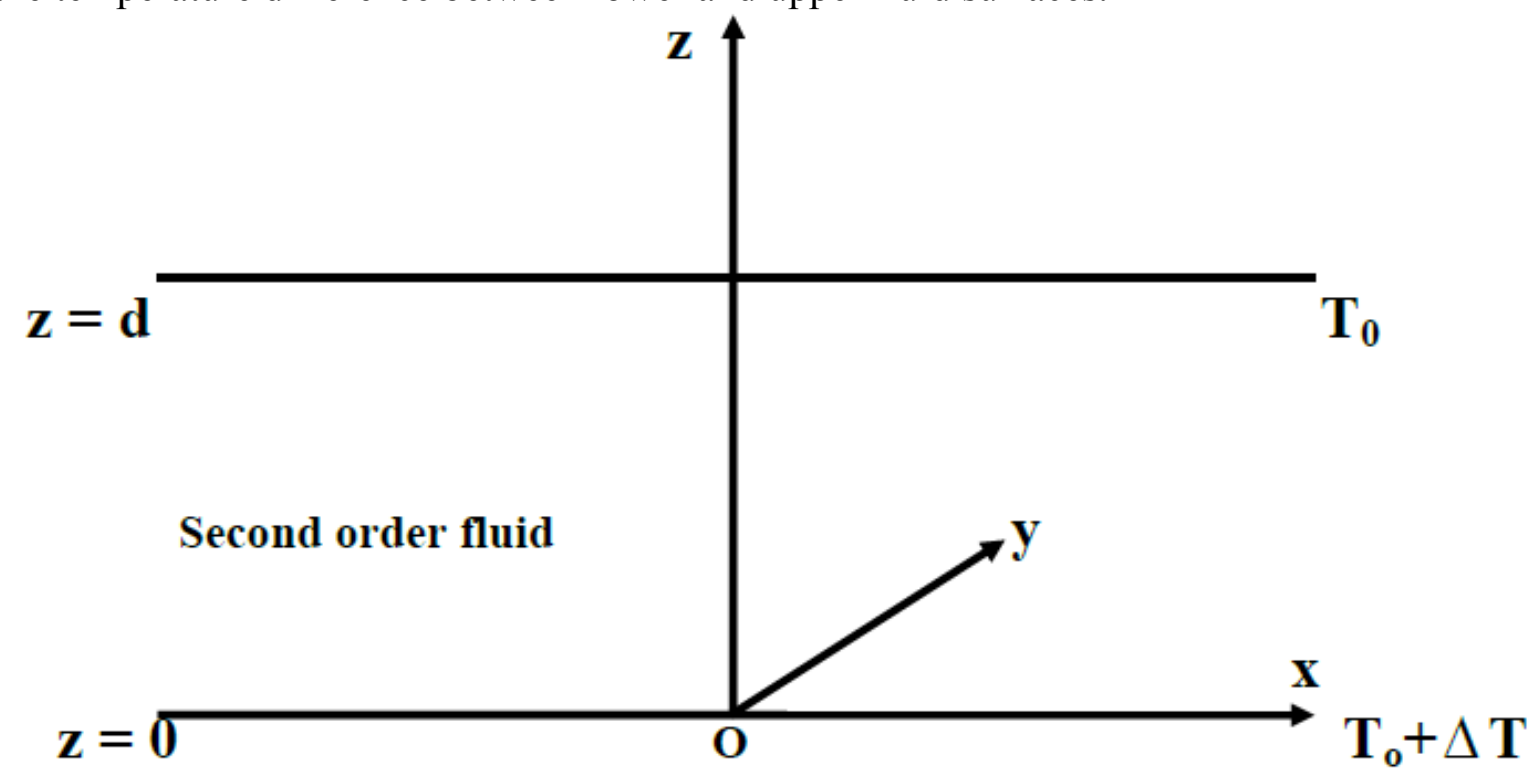

Fig. 1. Physical Configuration for a Rayleigh - Bènard Situation in a Second Order Fluid

The governing equations for the Rayleigh-Bènard situation in a Boussinesquian second order fluid are,

\section{Continuity Equation:}

$$
\nabla \cdot \vec{q}=0
$$

Linear Momentum Equation:

$$
\rho_{0}\left[\frac{\partial \vec{q}}{\partial t}+(\vec{q} \cdot \nabla) \vec{q}\right]=-\nabla p-\rho g \hat{k}+\mu_{1}\left[\nabla^{2} \vec{q}\right]+\mu_{2}\left[\frac{\partial}{\partial t}\left(\nabla^{2} \vec{q} \cdot \nabla\right) \vec{q}+(\vec{q} \cdot \nabla) \nabla^{2} \vec{q}\right],
$$

Energy equation:

$$
\frac{\partial T}{\partial t}+(\vec{q} \cdot \nabla) T=-\nabla \cdot \vec{Q}_{1},
$$

Maxwell-Cattaneo Heat Flux Equation:

$$
\tau\left[\dot{\vec{Q}}_{1}+\vec{w}_{1} \times \vec{Q}\right]=-\vec{Q}_{1}-\kappa \nabla T,
$$

where, $\vec{w}_{1}=\frac{1}{2} \nabla \times \vec{q}$ 


\section{Equation of state:}

$$
\rho=\rho_{0}\left[1-\alpha\left(T-T_{0}\right)\right]
$$

where, $\vec{\imath}$ is the velocity, $p$ is the pressure, $T$ is the temperature, $\rho$ is the density, $\rho_{0}$ is the density at $T=T_{0}, \mu_{1}$ is the viscocity, $\mu_{2}$ is the elasticity, $g$ is the acceleration due to gravity, $\vec{L}_{1}$ is the heat flux velocity, $\tau$ is the constant relaxation time and $\alpha$ is the co-efficient of thermal expansion.

\section{Basic state equations:}

The basic state of the fluid is quiescent and is described by,

$$
\vec{q}_{b}=(0,0,0), p=p_{b}(z), \rho=\rho_{b}(z), T=T_{b}(z)+T^{\prime}, \vec{Q}_{1 b}=\left(0,0, Q_{b}(z)\right),
$$

Equations (2) to (5) in the basic state specified by equation (6) becomes

$$
\begin{aligned}
& -\left(\frac{d p_{b}}{d z}\right)-\rho_{b} g=0, \\
& \frac{d \vec{Q}}{d z}=0, \\
& \vec{Q}_{b}=-\kappa \frac{d T_{b}}{d z}, \\
& \rho_{b}=\rho_{0}\left[1-\alpha\left(T_{b}-T_{0}\right)\right],
\end{aligned}
$$

We apply infinite perturbations on the quiescent basic state and study the stability.

\section{LINEAR STABILITY ANALYSIS}

Let the basic state be disturbed by an infinitesimal thermal perturbation. We now have

$$
\vec{q}=\vec{q}_{b}+\vec{q}^{\prime}, \vec{w}=\vec{w}_{b}+\vec{w}^{\prime}, p=p_{b}+p^{\prime}, \rho=\rho_{b}+\rho^{\prime}, T=T_{b}+T^{\prime}, \vec{Q}_{1}=\vec{Q}_{1 b}+\vec{Q}_{1}^{\prime},
$$

The prime indicates that the quantities are infinitesimal perturbations and the subscript , , $\mathrm{b}^{\text {ee }}$ indicates basic state value.

Substituting (11) to equations (1) to (5) and using the basic state solutions, we get linearized governing equations with infinitesimal perturbation in the form,

$$
\begin{aligned}
& \nabla \cdot \vec{q}^{\prime}=0, \\
& \rho_{0}\left[\frac{\partial \vec{q}^{\prime}}{\partial t}\right]=-\nabla p^{\prime}-\rho^{\prime} g \hat{k}+\mu_{1}\left[\nabla^{2} \vec{q}^{\prime}\right]+\mu_{2}\left[\frac{\partial}{\partial t}\left(\nabla^{2} \vec{q}^{\prime}\right)\right], \\
& \frac{\partial T^{\prime}}{\partial t}+w \frac{d T_{b}}{d z}=-\nabla \cdot \vec{Q}^{\prime}, \\
& \left(1+\tau \frac{\partial}{\partial t}\right) \vec{Q}^{\prime}=-\frac{\tau}{2} \frac{\Delta T}{d}\left[\frac{\partial \vec{q}^{\prime}}{\partial z}-\nabla w^{\prime}\right]-\kappa \nabla T^{\prime}, \\
& \rho^{\prime}=-\rho_{0} \alpha T^{\prime},
\end{aligned}
$$


Operating divergence through equation (15) and using (14) we get,

$$
\left(1+\tau \frac{\partial}{\partial t}\right)\left(\frac{\partial T^{\prime}}{\partial t}\right)=\left(1+\tau \frac{\partial}{\partial t}\right)\left(w^{\prime} \frac{\Delta T}{d}\right)-\frac{\tau}{2} \frac{\Delta T}{d} \nabla^{2} \cdot w^{\prime}+\kappa \nabla^{2} T^{\prime},
$$

The perturbation equations (13), (14), (15) and (17) are non-dimensionalised using the following definitions,

$$
\left(x^{*}, y^{*}, z^{*}\right)=\left(\frac{x}{d}, \frac{y}{d}, \frac{z}{d}\right), \vec{q}^{*}=\frac{\vec{q}^{\prime}}{\chi / d}, t^{*}=\frac{t}{d^{2} / \chi}, T^{*}=\frac{T^{\prime}}{\Delta T}, \vec{Q}_{1}^{*}=\frac{\vec{Q}_{1}}{\Delta T},
$$

Using equation (16) in eqauation (13), operating curl twice on the resulting equation and nondimensionalizing the resulting equation, also equation (13) and equation (17), and writing the kth component, we get

$$
\begin{aligned}
& \frac{1}{P_{r}} \frac{\partial}{\partial t}\left(1-Q \nabla^{2}\right)\left(\nabla^{2} w\right)=R\left(\nabla_{1}^{2} T\right)+\nabla^{4} w \\
& (1+2 C \sigma) \sigma T=(1+2 C \sigma) w-C \nabla^{2} w+\nabla^{2} T
\end{aligned}
$$

Where the asterisks have been dropped for simplicity and the non-dimensional parameters,

$$
\begin{aligned}
& \operatorname{Pr}=\frac{v}{\chi} \\
& Q=\frac{\mu_{2}}{\rho_{o} d^{2}} \\
& R=\frac{\alpha g \Delta T d^{3}}{v \chi} \\
& \mathrm{C}=\frac{\tau \chi}{2 \mathrm{~d}^{2}}
\end{aligned}
$$

(Prandtl number),

(Viscoelastic parameter),

(Rayleigh number),

(Cattaneo number)

\section{NORMAL MODE ANALYSIS}

The infinitesimal perturbations $\mathrm{W}$ and $\mathrm{T}$ are assumed to be periodic and since these permits a normal mode solution in the form

$$
[W, T]=[W(z), T(z)] e^{i(b x+m y)+\sigma t},
$$

Where 1 and $m$ are the horizontal wave number and $\sigma$ is the frequency. Substituting (21) in (19) and (20), we get

$$
\begin{aligned}
& \qquad \frac{\sigma}{P_{r}}\left(1-\vec{Q}\left(D^{2}-a^{2}\right)\left(D^{2}-a^{2}\right) W\right]=-R a^{2} T+\left(D^{2}-a^{2}\right)^{2} W, \\
& \\
& (1+2 C \sigma) \sigma T=(1+2 C \sigma) W-C\left(D^{2}-a^{2}\right) W+\left(D^{2}-a^{2}\right) T, \\
& \text { where }=\frac{d}{d z}
\end{aligned}
$$


The above equations are solved using the general boundary conditions on velocity and temperature.

$$
\left.\begin{array}{ll}
w=\frac{d^{2} w}{d z^{2}}-D_{i l} \frac{d w}{d z}=0, \frac{d T}{d z}-B_{i l} T=0, & \text { at } z=0 \\
w=\frac{d^{2} w}{d z^{2}}-D_{i u} \frac{d w}{d z}=0, \frac{d T}{d z}-B_{i u} T=0 & \text { at } z=1
\end{array}\right\} \text {, }
$$

where $D_{i l}$ and $D_{i u}$ are the Darcy numbers for lower and upper boundaries and $B_{i l}$ and $B_{i u}$ are the Biot numbers for lower and upper boundaries. We now apply Galerkin method to equations (22) and (23) to find the critical eigen value. Multiplying the equation (22) by $\mathrm{W}$ and equation (23) by $\mathrm{T}$, integrating the resulting equation by parts w.r.t. $\mathrm{z}$ between 0 and 1 and taking $\mathrm{W}=\mathrm{AW}_{1}$ and $\mathrm{T}=\mathrm{BT}_{1}$, where $\mathrm{A}$ and $\mathrm{B}$ are constants with $\mathrm{W}_{1}$ and $\mathrm{T}_{1}$ trial functions.

This procedure yields the following expression for Rayleigh number $\mathrm{R}$.

$$
R=\frac{\left(M_{1}-\frac{\sigma}{P_{r}} M_{2}+\frac{\sigma Q}{P_{r}} M_{1}\right)\left((1+2 C \sigma) \sigma M_{5}-M_{3}\right)}{a^{2} M_{6}\left((1+2 C \sigma) M_{6}-C M_{4}\right)},
$$

where,

$$
\begin{aligned}
& M_{1}=\left\langle W_{1}\left(D^{2}-a^{2}\right)^{2} W_{1}\right\rangle, M_{2}=\left\langle W_{1}\left(D^{2}-a^{2}\right) W_{1}\right\rangle, M_{3}=\left\langle T_{1}\left(D^{2}-a^{2}\right) T_{1}\right\rangle, \\
& M_{4}=\left\langle T_{1}\left(D^{2}-a^{2}\right) W_{1}\right\rangle, M_{5}=\left\langle T_{1}^{2}\right\rangle, M_{6}=\left\langle W_{1} T_{1}\right\rangle,
\end{aligned}
$$

Substituting $\sigma=i \omega$ in equation (25) and separating real and imaginary parts we get,

$$
R=\frac{\left[X_{1} X_{3}-2 C \omega^{2} M_{6} X_{2}+2 C \omega^{2} M_{1} M_{5} M_{6}\right]+i \omega\left[X_{3}\left(X_{2}+M_{1} M_{5}\right)-2 C M_{6} X_{1}\right]}{a^{2} M_{6} X_{3}},
$$

where,

$$
\begin{aligned}
& X_{1}=-M_{1}\left(2 C \omega^{2} M_{5}+M_{3}\right)-\omega^{2}\left(\frac{Q}{\operatorname{Pr}} M_{1}-\frac{1}{\operatorname{Pr}} M_{2}\right) M_{5}, \\
& X_{2}=\left(\frac{Q M_{1}}{\operatorname{Pr}}-\frac{1}{\operatorname{Pr}} M_{2}\right)\left(2 C \omega^{2} M_{5}+M_{3}\right), \\
& X_{3}=M_{6}-C M_{4},
\end{aligned}
$$

Equating imaginary part of equation (26) to zero, solving for $\omega^{2}$ we get,

$\omega^{2}=\frac{\left(M_{6}-C M_{4}\right)\left[\frac{M_{3}}{\operatorname{Pr}}\left(Q M_{1}-M_{2}\right)-M_{1} M_{5}\right]-2 C M_{1} M_{3} M_{6}}{4 C^{2} M_{1} M_{5} M_{6}+\frac{2 C M_{5} M_{6}}{\operatorname{Pr}}\left(Q M_{1}-M_{2}\right)-\frac{2 C M_{5}}{\operatorname{Pr}}\left(Q M_{1}-M_{2}\right)\left(M_{6}-C M_{4}\right)}$,

$$
R=\frac{X_{1} X_{3}-2 C \omega^{2} M_{6} X_{2}+2 C \omega^{2} M_{1} M_{5} M_{6}}{a^{2} M_{6} X_{3}},
$$


The trial functions for $W_{1}(z)$ and $T_{1}(z)$ satisfying the boundary conditions are given by,

$$
\begin{aligned}
& W_{1}=z^{4}+A z^{3}+B z^{2}+E z, \\
& T_{1}=z^{2}+F z+G,
\end{aligned}
$$

where,

$$
\begin{array}{ll}
A=\frac{-2\left(12+5 D_{i l}+3 D_{i u}+D_{i l} D_{i u}\right)}{12+4 D_{i l}+4 D_{i u}+D_{i l} D_{i u}} & , B=\frac{D_{i l}\left(6+D_{i u}\right)}{12+4 D_{i l}+4 D_{i u}+D_{i l} D_{i u}}, \\
E=\frac{2\left(6+D_{i u}\right)}{12+4 D_{i l}+4 D_{i u}+D_{i l} D_{i u}} & \quad F=\frac{B_{i l}\left(2-B_{i u}\right)}{B_{i l} B_{i u}+B_{i u}-B_{i l}}, \\
G=\frac{2-B_{i u}}{B_{i l} B_{i u}+B_{i u}-B_{i l}} &
\end{array}
$$

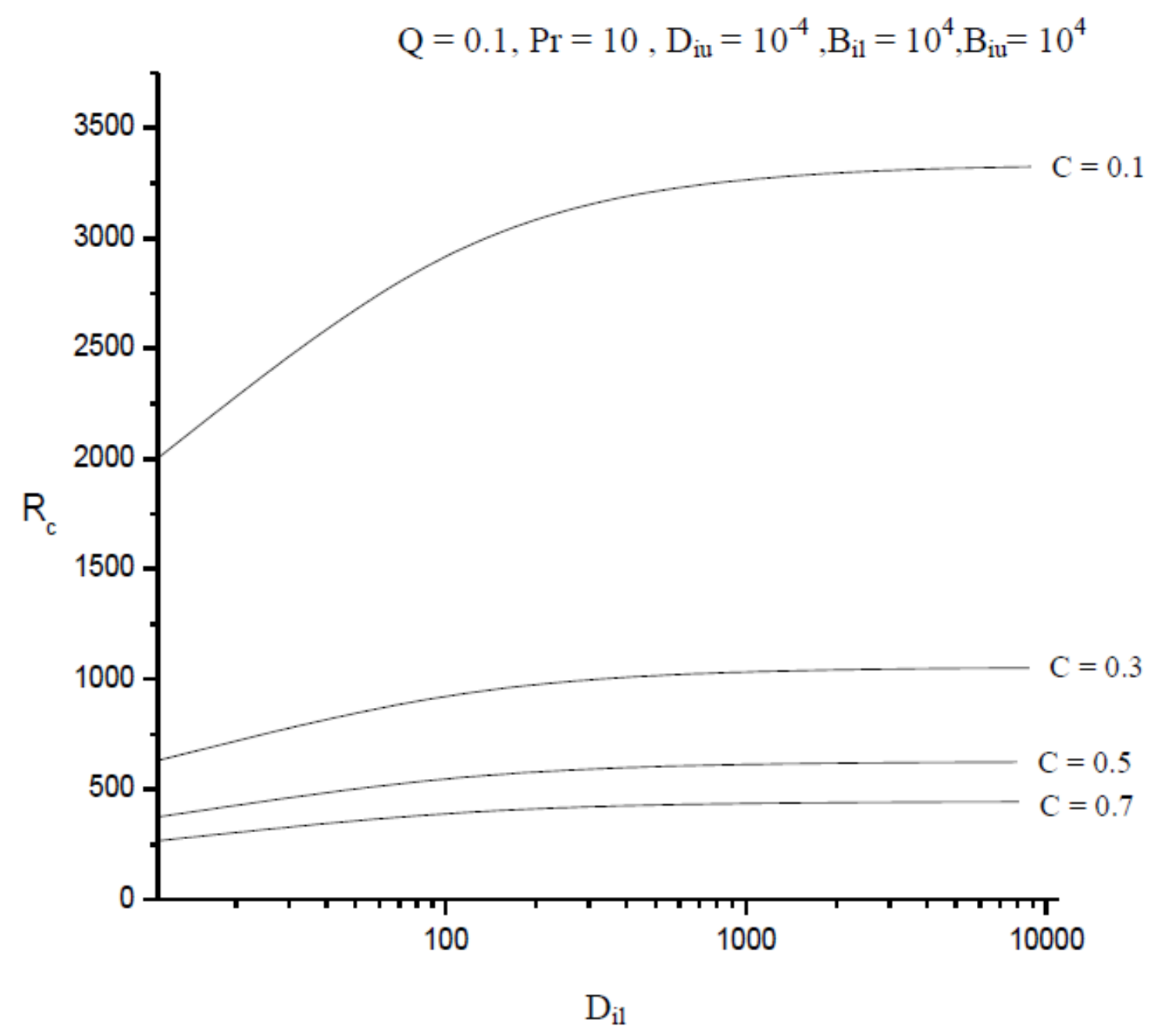

Fig. 2: Plot of critical Rayleigh number Rc Vs Darcy number Dil, for different values of Cattaneo number $C$. 


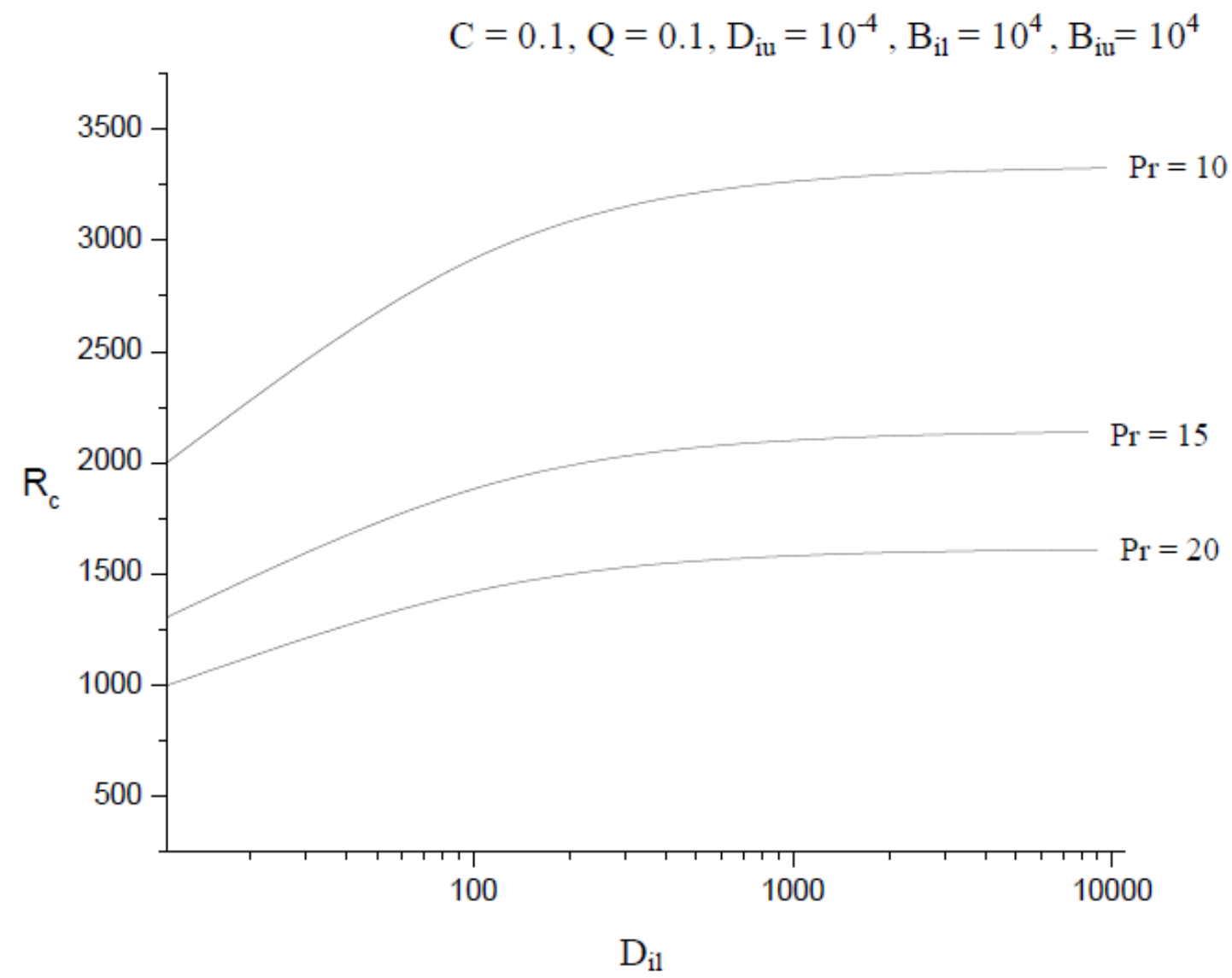

Fig. 3: Plot of critical Rayleigh number Rc Vs Darcy number Dil, for different values of Prandtl number Pr.

$$
\mathrm{C}=0.1, \operatorname{Pr}=10, \mathrm{D}_{\mathrm{iu}}=10^{4}, \mathrm{~B}_{\mathrm{il}}=10^{-4}, \mathrm{~B}_{\mathrm{iu}}=10^{-4}
$$

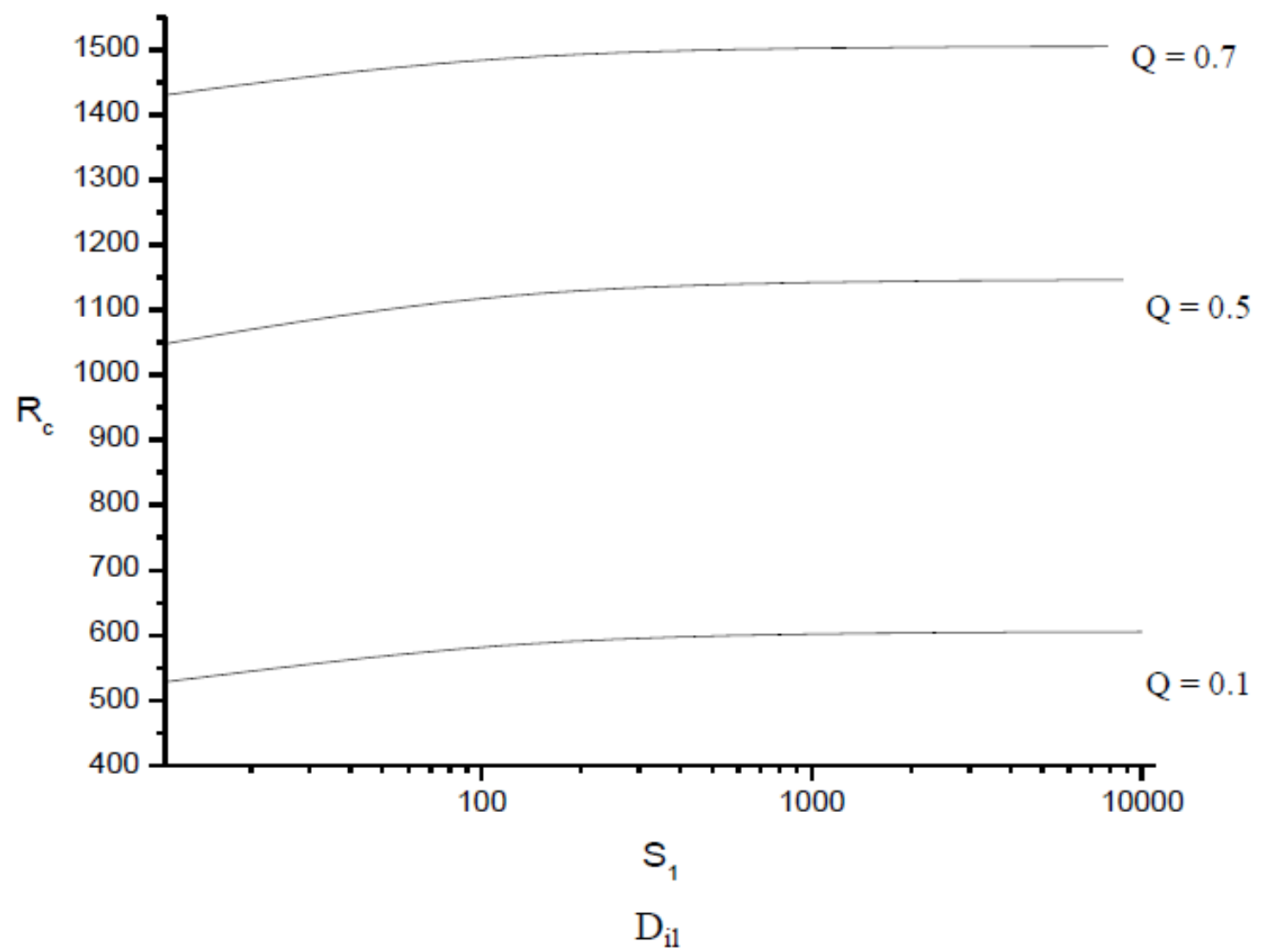

Fig. 4: Plot of critical Rayleigh number Rc Vs Darcy number Dil, for different values of Q. 


\section{RESULTS AND DISCUSSIONS :}

In this problem, we study the onset of Rayleigh-Bènard convection in Coleman-Noll fluid by replacing the classical parabolic heat equation by a non-classical heat flux using general boundary conditions on velocity and temperature at the upper and lower walls.

From figures (2) to (4) the following results are obtained :

1. The increase in Cattaneo number $\mathrm{C}$ decreases the $\mathrm{Rc}$, the effect of Cattaneo number is to destabilize the system. Increase in Cattaneo number leads to narrowing of the convection cells and thus lowering of the critical Rayleigh number. It is also observed from figures that influence of Cattaneo number is determined for small values because the convection cells have fixed aspect ratio.

2. The increase in Prandtl number $\mathrm{Pr}$, decreases the Rc , the effect of $\mathrm{Pr}$ is to destabilize the system.

3. The increase in viscoelastic parameter Q (Second order effect) increases the Rc , thus stabilize the system.

4. It has been observed that the critical Rayleigh number in the case of rigid-rigid surfaces is higher than those of rigid-free surfaces and free-free surfaces as expected from physical grounds. Further the critical Rayleigh numbers in the case of isothermal boundaries are greater than those of adiabatic boundaries .

5. We can conclude $R_{c}^{H H E}<R_{c}^{P H E}$ where HHE -Hyperbolic heat equation, PHE - Parabolic heat equation.

\section{CONCLUSION:}

1. The non-classical Maxwell-Cattaneo heat flux law involves a wave type heat transport and does not suffer from the physically unacceptable drawback of infinite heat propagation speed. The classical Fourier flux law over predicts the critical Rayleigh number compared to that predicted by the non-classical law. Over stability is the preferred mode of convection.

2. The study of the problem with general boundary conditions for velocity and temperature clearly indicates that the results of the previous studies for different boundary combinations with $\mathrm{C}=0$ are obtained as limiting cases of the present study.

3. From the mathematical point of view it is important to note that the general formulation of the problem suggests the need to give up the tackling of limiting cases in isolation. In addition, the general formulation brings the theoretical study more closer to reality compared to limiting cases.

\section{Acknowledgement:}

The authors would like to thank the management of Christ University for their support in completing this work.

\section{REFERENCES}

[1] Coleman B. D, Noll W., 1960, “An Approximation Theorem For Functionals With Applications In Continuum Mechanics”, Arch. Rational Mech. Anal., Vol. 6, pp. 355.

[2] Kaloni P. N., 1989, “ Some Remarks On Useful Theorems For Second-Order Fluids”, J. NonNewt. Fluid Mech., Vol. 31, pp. 115.

[3] Siddheshwar. P. G. and Srikrishna, C. V. (2002) "Unsteady non-linear convection in a second order fluid", Int. J. Non-linear Mech., Vol. 37, pp. 321.

[4] Siddeshwar. P. G, Sekhar G. N, Jayalatha G. (2010), "Effect of time period vertical oscillations of the Rayleigh- Bènard system on non-linear convection in viscoelastic liquids," J. Non Newtonian fluid Mech., Vol. 165, pp. 1412. 
[5] Sekhar G. N , Jayalatha G. (2010), "Elastic effect on Rayleigh Bènardin liquid in temperature dependent viscocity," Int. Journal of thermal sciences, Vol. 49, pp. 67.

[6] Lindsay K. A. and Straughan, B. (1978) "Acceleration waves and second sound in a perfect fluid”, Arch. Rational Mech. Anal. Vol. 68, 53.

[7] Straughan B. and Franchi F. (1984) "Benard convection and the Cattaneo law of heat conduction", Proc. of Roy. Soc. Of Edi. Vol. 96A, 175.

[8] Lebon G. and Cloot A. (1984) "Benard-Marangoni instability in a Maxwell-Cattaneo fluid", Phy. Let. Vol 105 A, 361.

[9] Siddheshwar P. G. (1999) "Rayleigh Benard convection in a second order Ferromagnetic fluid with second sound". Proc. VIII Asian Cong., Fluid Mech. China, Dec. 6-10, 631.

[10] Siddheshwar P. G. and Pranesh S. (1998) "Effects of a Non-Uniform Basic Temperature Gradient on Rayleigh-Bénard Convection in a Micropolar Fluid", International Journal of Engineering Science, Vol. 36, No. 11, September, pp. 1183.

[11] Pranesh S. and Kiran R. V. (2010), "Study of Rayleigh-Bènard Magneto convection in a micropolar fluid with Maxwell Cattaneo law”, Applied Mathematics, Vol. 1, pp. 470. 